\title{
Asymptomatic UTI in Pregnancy Women Attending at Ante- Natal Care Clinic at Minia Obstetrics University Hospitals
}

\author{
Lamiaa H. Abdel-Hak, Omima M. M and Salwa H. Fawzy. \\ Department of Clinical Pathology, Faculty of Medicine, Minia University, Egypt
}

\begin{abstract}
Background: Urinary tract infections (UTI) during pregnancy issued by inappropriate laboratory investigations situate women at high risk of several complications. Untreated and neglected asymptomatic UTI may lead to pyelonephritis, preterm birth, low birth weight, etc. Urine culture is used to confirm the diagnosis of UTI. Objectives: This work aims to find the prevalence of asymptomatic UTI and to establish antimicrobial drug susceptibility of the pathogens resulting asymptomatic UTI in pregnant women attending at ante-natal care clinic at Minia Obstetrics University Hospitals. Methods: The urine samples were collected through mid-stream clean catch method from 90 pregnant women of age15-45 years visiting for ante-natal clinic. All the urine samples were subjected to biophysical, biochemical, microscopic, and microbiological analysis. The prevalence of UTI in three trimesters and in different age groups was obtained together with antimicrobial drug susceptibility tests. Results: On overall $(38.8 \%)$ of urine samples revealed with significant bacteriuria and considered as the asymptomatic UTI. On One way ANOVA test, there was significant $(\mathrm{P}<0.001)$ prevalence of asymptomatic UTI when compared among different trimesters, higher prevalence of asymptomatic UTI being in the third trimester (84\%). Prevalence of culture positivity with respected age was maximum between the age group of 25-30 (41.4\%). Escherichia coli were the most implicated organism (52.8\%) followed by Klebsiella pneumonae (18.6\%). The gramnegative bacteria (E. coli and K. pneumonae) were more sensitive against Amikacin, Gentamycin, and Nitrofurantointhan. Other drugs whereas; gram positive bacteria had high susceptibility with Vancomycin. Conclusion: There was remarkable incidence of asymptomatic UTI among the pregnant women in the study region; therefore, screening of urine of all pregnant women is very important and should be included in the routine antenatal checkups to detect the symptomatic UTI to prevent the maternal and obstetric complications in them.
\end{abstract}

Keywords: Asymptomatic UTI, Pregnancy, Prevalence of UTI.

\section{Introduction}

Asymptomatic bacteriuria (ASB) is defined as the presence of more than 100,000 colonies of a single bacterial species per milliliter of urine, cultured from midstream sample in the absence of declared symptoms (Kim et al., 2018).

Asymptomatic bacteriuria is common in women and increases in prevalence with age and/or sexual activity. The prevalence of asymptomatic bacteriuria in pregnancy varies form 4$7 \%$ is similar to that observed in non-pregnant women (Wingert et al., 2017).

Although pyelonephritis develops in $20-40 \%$ of pregnant women with untreated asymptomatic bacteriuria, up to $80 \%$ of cases could be prevented by treating asymptomatic bacteriuria. It has been clearly established that untreated bacteriuria can have serious implications for both mother and fetus (Patel et al., 2018).

Screening for asymptomatic bacteriuria is a standard of obstetrical care and is included in most antenatal guidelines. While several rapid screening tests have been evaluated, none perform adequately to replace urine culture for detecting a symptomatic bacteriuria (Khanum et al., 2017).

Routine urine culture cannot be carried out for every pregnant woman during her antenatal checkups presumably due to poor health education, high cost and time duration for culture result (usually2-3days). Rather many clinicians pick up for the strip urinalysis method for pregnant women which poorly 
quantify the grade of infection in them (Sinawe et al., 2020).

\section{The aim of work:}

To evaluate the prevalence of asymptomatic UTI and to establish antimicrobial drug susceptibility of the pathogens resulting asymptomatic UTI in pregnant women attending at ante-natal care clinic at Minia Obstetrics University Hospitals.

\section{Subjects and Methods}

The present study has been conducted on 70 consecutive female pregnant women, their ages ranged from 19 to 45 years old attending at ante-natal clinic of Gynecology \& Obstetrics Department of Minia University Hospital, through the period July 2019 to December 2019. In addition to 20 apparently healthy pregnant volunteers who served as a control group of matched age ranged from (19 - 45) and gestational age.

All patients were subjected to full history taking, general examination and laboratory investigations including $\mathrm{HB}$, RBCs, TLC, absolute neutrophilic count, renal functions tests, random blood glucose level, serum CRP, urine analysis and urine culture.

\section{Statistical analysis}

Data were statistically analyzed using the IBM SPSS 20.0 package software. Analysis of variance (ANOVA) was used for comparison between independent groups for parametric data followed by LSD post hoc test to assess intergroup differences.

Kruskal Wallis test for non-parametric quantitative data followed by Mann Whitney test to compare each two groups and the Chi-square test or Fisher's exact test were used to compare categorical variables. A $P$ value of 0.05 or less was considered significant.

\section{Results}

On overall (38.8\%) of urine samples revealed as asymptomatic UTI. There was significant $(\mathrm{P}<0.001)$ prevalence of asymptomatic UTI when compared among different trimesters, higher prevalence of asymptomatic UTI being in the third trimester (84\%).
Prevalence of culture positivity with respected age was maximum between the age group of 25-30 (41.4\%). Escherichia coli were the most implicated organism (52.8\%) followed by Klebsiella pneumonae (18.6\%).

DM, preeclampsia and Premature rapture of membrane have no statistically significant difference $(\mathrm{P}$-value $=0.303,0.322,0.204)$ respectively.

The gram-negative bacteria (E. coli and K. pneumonae) were more sensitive against Amikacin, Gentamycin, and Nitrofurantointhan. Other drugs whereas; gram positive bacteria had high susceptibility with Vancomycin.

\section{Discussion}

In pregnancy, there are hormonal, metabolic and mechanical factors that provide conditions suitable for microbial growth in urine and facilitate a greater incidence of ASB in pregnant women, if neglected with no treatment, serious complications occurred and exacerbated maternal and neonatal morbidity. Thus, antenatal screening for asymptomatic bacteriuria is a must.

Dipstick analysis is a rapid screening test to diagnose UTI and showed high significant difference but it is not very specific to UTI as it may misdiagnose UTI with other renal conditions as stones and cancer.

Urine culture is the gold standard test to diagnose UTI but it is expensive, taking 72 hours, requiring laboratory resources for appropriate result. So a screening test or marker is needed for rapid diagnosis of UTI in pregnancy and to start empirical treatment of antibiotics.

E. coli was found to be predominant organism to cause urinary tract infection followed by Klebsiella.

Amikacin, Gentamycin, and Nitrofurantointhan are the most effective drugs against gram negative bacteria while Vancomycin has remarkable results against gram positive pathogens. 


\section{References}

1. Khanum, S., Ahmed, J.U. and Khanam, K., 2017: Bacterial Etiology, Antibiotic Sensitivity Pattern and Risk Factors for Asymptomatic Bacteriuria during Pregnancy: Experience in a Tertiary Care Hospital. BIRDEM Medical Journal, 6(2), pp.79-83.

2. Kim, Sharon J, Parikh P, King A.N and Marnach, M.L., 2018: Asymptomatic Bacteriuria in Pregnancy Complicated by Pyelonephritis Requiring Nephrectomy. Case reports in obstetrics and gynecology, 2018.
3. Patel, K., Aftandilians, T., GittensWilliams, L. and Apuzzio, J., 2018: Outcomes of anemic patients with acute pyelonephritis during pregnancy. American Journal of Obstetrics and Gynecology, 218(1), p.S526.

4. Wingert, A., Pillay, J., Featherstone, R., Gates, M., Sebastianski, M., Shave, K., Vandermeer, B., Hartling, L., Biringer, A., Smaill, F. and Doull, M (2017): Screening for asymptomatic bacteriuria in pregnancy: systematic review and meta-analysis, p1244.

5. Sinawe, H. \& Casadesus, D. (2020): Urine Culture. StatPearls. StatPearls Publishing. 